\title{
An instantaneous spatiotemporal model to predict a bicyclist's Black Carbon exposure based on mobile noise measurements.
}

Luc Dekonincka)

Dick Botteldooren $b$ )

Information Technology, Acoustics Group, Ghent University

St-Pietersnieuwstraat 41, 9000 Ghent, Belgium ${ }^{\mathrm{a}, \mathrm{b}}$

Luc Int Panis $\mathrm{s}_{\mathrm{c}, \mathrm{d}}$

Flemish Institute for Technological Research (VITO), Boeretang 200, 2400 Mol, Belgiumc

Transportation Research Institute (IMOB), Hasselt University, Wetenschapspark 5 bus 6, 3590 Diepenbeek, Belgium ${ }^{\mathrm{d}}$

\begin{abstract}
Several studies have shown that a significant amount of daily air pollution exposure, in particular black carbon (BC), is inhaled during trips. Assessing this contribution to exposure remains difficult because on the one hand local air pollution maps lack spatio-temporal resolution, at the other hand direct measurement of particulate matter concentration remains expensive. This paper proposes to use in-traffic noise measurements in combination with geographical and meteorological information for predicting BC exposure during commuting trips. Mobile noise measurements are cheaper and easier to perform than mobile air pollution measurements and can easily be used in participatory sensing campaigns.
\end{abstract}

The uniqueness of the proposed model lies in the choice of noise indicators that goes beyond the traditional overall A-weighted noise level used in previous work. Noise and BC exposures are both related to the traffic intensity but also to traffic speed and traffic dynamics. Inspired by theoretical knowledge on the emission of noise and $\mathrm{BC}$, the low frequency engine related noise and the difference between high frequency and low frequency noise that indicates the traffic speed, are introduced in the model. In addition, it is shown that splitting BC in a local and a background component significantly improves the model. The coefficients of the proposed model are extracted from 200 commuter bicycle trips. The predicted average exposure over a single trip correlates with measurements with a Pearson coefficient of 0.78 using only four parameters: the low frequency noise level, wind speed, the difference between high and low frequency noise and a street canyon index expressing local air pollution dispersion properties.

\section{Highlights}

- We performed combined black carbon and traffic noise measurements by bicycle.

- After correcting for the black carbon background concentrations a successful model is build.

- Personal noise measurements can be used as a proxy for black carbon exposure for bicyclists.

Keywords: Black Carbon, Vehicle Noise, Personal exposure, Bicyclists, Traffic

\footnotetext{
a) email: luc.dekoninck@intec.ugent.be (corresponding author, tel +32 926499 95)

b) email: dick.botteldooren@intec.ugent.be

c) email: luc.intpanis@vito.be; luc.intpanis@uhasselt.be
} 
Exposure to particulate matter is currently regulated in PM standards, that only distinguish between the size of the particles (PM10, PM2.5, ...) and between the composition and thus origin of the particulate matter. The soot fraction, Black Carbon (BC) is the part of the PM directly related to combustion processes. Recent evidence, summarized by the world health organization (WHO), documents the relevance of $\mathrm{BC}$ for evaluating traffic related health effects (WHO Europe, 2102). The first epidemiological results suggest health effects that are up to 10 times higher than a similar evaluation based on PM10. Further research into health effects is hampered by the difficulty to measure or model $\mathrm{BC}$ concentrations. An important reason for this is the stronger spatial variability than for example PM10 variability. In addition, efforts are now made to standardize $\mathrm{BC}$ measurements as a first step to including $\mathrm{BC}$ in the set of official air pollution standards. For these reasons, this paper takes a closer look at an innovative way to predict a bicyclist's Black Carbon exposure.

Large personal exposure measurement campaigns proof the relevance of the in-traffic exposure contribution (Dons et al., 2011, 2012). Technology for mobile air pollution measurements is however scarce and expensive. On the one hand, cheap implementations do not meet quality requirements, at the other hand high quality equipment is often highly demanding on the operator (e.g. changing filters or liquids, limited portability). In contrast, mobile noise measurements can be done with low intrusive measurement equipment like dosimeters and new mobile technologies. Mobile noise measurements are a popular theme in noise exposure modeling (Eisenman et al., 2009; Kanjo et al., 2010, Maissonneuve et al., 2009). Noise levels are strongly related to traffic related air pollution levels and might be a good proxy to model personal air pollution exposure (Dekoninck et al., 2012; Can et al., 2010, 2011; Eisenman et al. 2009, Foraster et al., 2011). Since in-traffic personal air pollution exposure is a major component of the total personal diurnal exposure and diurnal activity patterns are very divers within the population, epidemiology would benefit from including exposure differences due to different activity patterns when investigating the health effects of air pollution (von Klot et al., 2011; Dons et al., 2012). The use of a proxy which is easy to monitor could result in improved personal exposure estimates on larger population samples at a reasonable cost.

In this paper the focus is on the personal exposure of cyclists. Numerous efforts have been made to quantify the exposure and health effects of cycling in dense traffic since the current trends in sustainable 
mobility focus on establishing modal shifts towards biking and walking (de Nazelle et al., 2011; Int Panis et al., 2010; Berghmans et al., 2009; de Hartog et al., 2010). Exposure of cyclists is directly influenced by the distance to the local traffic, strongly related to local traffic conditions (congestion, traffic lights etc) and highly influenced by meteorological conditions. The strong effects of built-up areas on air pollution dispersion also influence the exposure of cyclists. The local traffic and its properties is an important contributor to bicyclist's exposure. Since cyclists often travel along low-traffic-density roads however, there is in general no traffic data available from either counting loops or traffic models. The most important exposure parameter is therefore unknown in most studies. Local traffic on low density roads is also highly variable in both space and time. A suitable traffic description should reflect these short-term effects with an adequate spatial and temporal resolution. Mobile noise exposure therefore has the potential to become this key indicator to predict the local component of traffic related air pollution exposure.

Based on theoretical aspects of traffic dynamics, the relationship between noise and particulate matter emission and the potential to extract one from the other have been discussed earlier (Dekoninck et al. 2012). The focus there was on the selection of noise descriptors correlating best with vehicular particulate matter emissions for typical traffic dynamics. However, noise exposure close to the source is not strongly influenced by the meteorological condition while air pollution is strongly affected by the meteorological conditions. This is one of the reasons why the average of repeated noise exposure measurements will converge faster compared to repeated air pollution exposure measurements. So, if mobile noise measurements are proven to be a valid proxy for air pollution exposure, fewer measurements will be needed to predict the personal exposure at a higher spatial and temporal resolution.

To establish an instantaneous relationship between noise exposure and air pollution exposure meteorological conditions have to be taken into account. A major concern is the influence of long term meteorology and long distance air pollution transport on the background concentrations influencing the actual personal exposure. In this paper two major research questions are addressed: (1) is a prediction model for BC exposure improved by separating out long term variations in the background concentration; (2) can the instantaneous local Black Carbon exposure be predicted based on instantaneous local noise exposure and meteorological conditions. For the latter, the question how to derive the local traffic dynamics that influence the instantaneous Black Carbon exposure from noise measurements, is addressed. Section 2 will address the 
methodology including the definition of the models and the noise exposure parameters. Section 3 gives the results of the models and the model validations. Results are discussed in section 4.

\section{Methodology}

\subsection{Measurement equipment and setup}

The experimental setup contains a basic GPS (in a HTC Desire smart phone), a Type 1 Noise Level Meter (Svantek 959) and a micro-aethalometer (Model AE51 MageeScientific, 2009) to measure Black Carbon. The measurements were performed while commuting by bicycle from the villages to the west of Ghent (Belgium) into the city center, thus covering the sparsely build areas in the villages, the city center, open recreational areas and natural reserves in between. A total of 209 biking trips were performed, covering a distance of 2300 kilometer, a total measurement time of 128 hours at an average speed of $18 \mathrm{~km}$ per hour. More than 75 $\mathrm{km}$ of distinct roads were sampled at least 3 times.

The details of the measurement setup, temporal resolution, preprocessing, meteorological data and the spatial mapping on aggregation points $\mathrm{p}_{\mathrm{x}}$ along the network with a spatial resolution of $50 \mathrm{~m}$ are available in the supplementary data. In this instantaneous model only one spatial attribute, the street canyon index $\operatorname{StCan}_{p_{x}}$ at aggregation point $\mathrm{p}_{\mathrm{x}}$ is included, identifying 'street canyon likeliness' of the built-up area along the trip trajectories. More detail on the calculation of $\operatorname{StCan}_{p_{x}}$ is available in the supplementary data.

\subsection{Black carbon, background and local contribution}

The $\mathrm{BC}$ exposure during a cycling trip consists of a contribution of local sources and a background contribution. The latter varies only little over a large area and can thus easily be obtained from a well-located fixed measurement station. The background contribution strongly depends on long-term meteorological conditions. The proposed model assumes that the dominant source of $\mathrm{BC}$ in the vicinity of the cyclist is the local traffic on the travelled road. An additive approach is used; the BC exposure is viewed as the sum of the background level $B C_{j, b g}$ during trip $\mathrm{j}$ and the "local" contribution $B C_{l o c}$. Similar procedures are used in exposure estimations where regional and local scale models are added to estimate personal exposure (Isakov et al., 2009). The available background measurements are averaged concentrations over half an hour (see supplementary data). Subtracting a fixed measurement with a temporal resolution of a half hour with mobile 
BC measurements sampled at 1 second time interval is not trivial. When sampling air pollution at a shorter time interval, concentrations below the "background" concentrations can be measured at some of the low traffic locations. Correcting for the background concentration could then result in negative local concentrations. The proposed models will use a logarithm of $\mathrm{BC}$ as an outcome variable because noise is also measured on a logarithmic scale and hence negative values cannot be allowed. For this reason the background concentration is not removed completely but replaced by a typical but constant low background concentration. The choice of this constant is not very critical since it will be added again to the model outcome and constants do not affect the model. This approach enables the dataset to retain spatial variation also for low density roads. Nevertheless a physical reference to the ambient concentration is useful, therefore the long term first quartile concentration over the whole measurement period is chosen, the resulting background $\mathrm{BC}$ concentration $Q 1\left(B C_{b g, l t}\right)$ is equal to $775 \mathrm{ng} / \mathrm{m} 3$. The relation between the measured $\mathrm{BC}_{\text {raw }}$ and the local contribution $B C_{l o c}$ for a location $i$ and during a trip $j$, can be written as:

$$
B C_{\text {raw }, i, j}=B C_{l o c, i, j}+\left(B C_{b g, j}-Q 1\left(B C_{b g, l t}\right)\right)
$$

Where $\mathrm{BC}_{\mathrm{bg}, \mathrm{j}}$ is the background concentration obtained from the continuous measuring station averaged over the whole duration of the trip j. At each aggregation points $p_{x}$ on the network, the arithmetic average of all $n$ measurements of trip $\mathrm{j}$ in this collection is calculated as:

$$
B C_{l o c, p_{x}, j}=\frac{1}{n} \sum_{\in P_{p x, j}} B C_{l o c, i, j}
$$

\subsection{Noise parameters and physical interpretation}

Details of noise parameter calculations are available in the supplementary data. For an even more detailed description of the theoretical and empirical relations of the noise parameters with the $\mathrm{BC}$ exposure the reader is referred to Dekoninck et al, 2012. The main arguments for selecting particular noise indicators to be included in the model, based on their physical and technical interpretations, are briefly reminded to the reader. Three noise parameters were included in the instantaneous modeling. The harmonized calculation method used for noise map calculations for the European Union (END Directive) separates the noise emission into an engine contribution and a rolling noise contribution. Engine noise is dominant in the low frequencies; rolling noise is dominant in the high frequencies at higher speeds. The two first parameters are directly related to these emission features. The first parameter $L_{O L F, p_{x}, j}(100-200 \mathrm{~Hz})$ describes the engine noise of the nearby traffic at point $\mathrm{p}_{\mathrm{x}}$ for trip j. High throttle increases the engine noise. $L_{H L F, p_{x}, j}(1000-2000 \mathrm{~Hz})$ is 
related to the rolling noise. The second parameter $L_{H F m L F, p_{x}, j}$ is the difference between high and low frequencies in the noise spectrum at point $\mathrm{p}_{\mathrm{x}}$ for trip j. High levels of $L_{H F m L F, p_{x}, j}$ indicate a relative stronger contribution of high frequencies, compared to low frequencies, indicating more rolling noise than engine noise due to the nearby traffic, hence traffic at higher speed. The third parameter $\left(L_{A e q}-L_{A m i n}\right)_{p_{x, j}}$ referred to as the short term dynamics of the noise at point $p_{\mathrm{x}}$ for trip $\mathrm{j}$, indicates the presence of noise events. If noise levels $\mathrm{L}_{\mathrm{Aeq}, 100 \mathrm{~ms}}$ within a single second change rapidly, the passing vehicles can individually be detected in the noise measurements. If $\left(L_{A e q}-L_{A m i n}\right)_{p_{x}, j}$ is low, noise levels are constant, indicating constant flow traffic with many sources in the vicinity.

\subsection{GAM modeling}

Generalized additive models (GAMs) are regression models where smoothing splines are used instead of linear coefficients for the covariates. This approach has been found to be particularly effective for handling the complex non-linearity associated with air pollution research (Dominici et al., 2002, Pearce et al., 2011). The additive model in the context of spatial exposure modeling can be written in the form:

$$
\log \left(B C_{p x, j}\right)=\sum_{z=1}^{n} s_{z}\left(v_{z, p x, j}\right)+\varepsilon_{x, j}
$$

Where $\mathrm{v}_{\mathrm{z}}$ is the $\mathrm{z}^{\text {th }}$ covariate evaluated for trip $\mathrm{j}$ at location $p_{x} ; s_{\mathrm{z}}\left(\mathrm{v}_{\mathrm{z}, \mathrm{px}, \mathrm{j}}\right)$ is the smooth function of $\mathrm{z}^{\text {th }}$ covariate, $\mathrm{n}$ is the total number of covariates, and $\varepsilon_{\mathrm{x}, \mathrm{j}}$ is the corresponding residual with $\operatorname{var}\left(\varepsilon_{\mathrm{x}, \mathrm{j}}\right)=\sigma^{2}$, which is assumed normally distributed. Smooth functions are developed through a combination of model selection and automatic smoothing parameter selection using penalized regression splines, which optimize the fit and try to minimize the number of dimensions in the model. The main advantage of GAM modeling is the possibility to adjust for non-linear relationships between the covariate and the outcome. The analysis was constructed using the GAM modeling function in the R environment for statistical computing (R development Core Team, 2009) with the package 'mgcv' (Wood, 2006).

Two modeling approaches will be discussed. The first option is to model the measurements on the basic aggregation level: one value for each parameter for each trip at each aggregation point $\mathrm{p}_{\mathrm{x}}$. This dataset contains about 37.700 records and will be referred to as the basic dataset (BDS). The second approach starts the modeling after aggregating the BDS to a dataset averaging the $\mathrm{BC}$ exposure for the classified parameters included in the aggregation models. Each of the retained parameters is classified according a set of predefined 
percentile classes (see supplementary data). For each of these two approaches the models will be evaluated for both the raw $\mathrm{BC}$ result $\left(\mathrm{BC}_{\text {raw }}\right)$ as for the local contribution $\mathrm{BC}_{\text {loc }}$ to assess the relevance of handling background concentrations separately.

\section{Results}

In this section, the following terminology will be used to refer to the different models that will be compared:

- $\quad \mathrm{BC}_{\mathrm{bg}}$ model: this model simply assumes that exposure during cycling trips equals concentrations measured at a regional background measurement station.

- $\quad \mathrm{BC}_{\text {raw }}$ model: uses GAM to obtain $\mathrm{BC}$ directly from noise, weather, and geometrical data.

- $\quad \mathrm{BC}_{\text {loc }}$ model: uses $\mathrm{GAM}$ to obtain $\mathrm{BC}_{\mathrm{loc}}$ and adds measured $\mathrm{BC}_{\mathrm{bg}}$ to obtain the overall concentration as shown in Eqs. (1).

- Aggregated $\mathrm{BC}_{\text {raw }}$ and $\mathrm{BC}_{\text {loc }}$ models: same as above but with predictive variables categorized in percentile classes.

\section{1 $\mathrm{BC}_{\text {loc }}$ and $\mathrm{BC}_{\text {raw }}$ model}

The $\mathrm{BC}_{\text {loc }}$ model is based on the BDS dataset and includes the three noise parameters, wind speed WS trip, $_{\text {, }}$ temperature Temptrip and street canyon index $\operatorname{StCan}_{p_{x}}$. The parameters of the GAM models are shown in Table 1. The quality of a GAM model and the relative strength of its parameters are described by the devience explained, the intercept, the F and p-value for each of its covariates. Since the number of data points in the models is large compared to the number of covariates, the degrees of freedom is large and the p-values are in general too small to be used to compare the covariates. The F-parameters present the relative strength of the covariates instead. In the $\mathrm{BC}_{\text {loc }}$ model the intercept and the $L_{O L F, p_{x}, j}$ have a similar strength; the wind speed is about half the strength of $L_{O L F, p_{x}, j}$. The plots of the splines created by the GAM modeling show the relation of the parameter to the outcome $\log \left(\mathrm{BC}_{\mathrm{loc}}\right.$ ) (Figure 1). In the $\mathrm{BC}_{\text {loc }}$ model the $L_{O L F, p_{x}, j}$ is the strongest component and has a linear relation with $\log \left(\mathrm{BC}_{\mathrm{loc}}\right) . L_{H F m L F, p_{x}, j}$ saturates for higher levels. $\log \left(\mathrm{BC}_{\mathrm{loc}}\right)$ decreases for high wind speeds. The street canyon effect is visible as a steady increase of the exposure with a higher street canyon index $\operatorname{StCan}_{p_{x}}$. The temperature is not very important, the steep upward trend is based on only a few 
samples; few trips were performed at temperatures above $20^{\circ} \mathrm{C} . \log \left(\mathrm{BC}_{\text {loc }}\right)$ increases strongly for very small
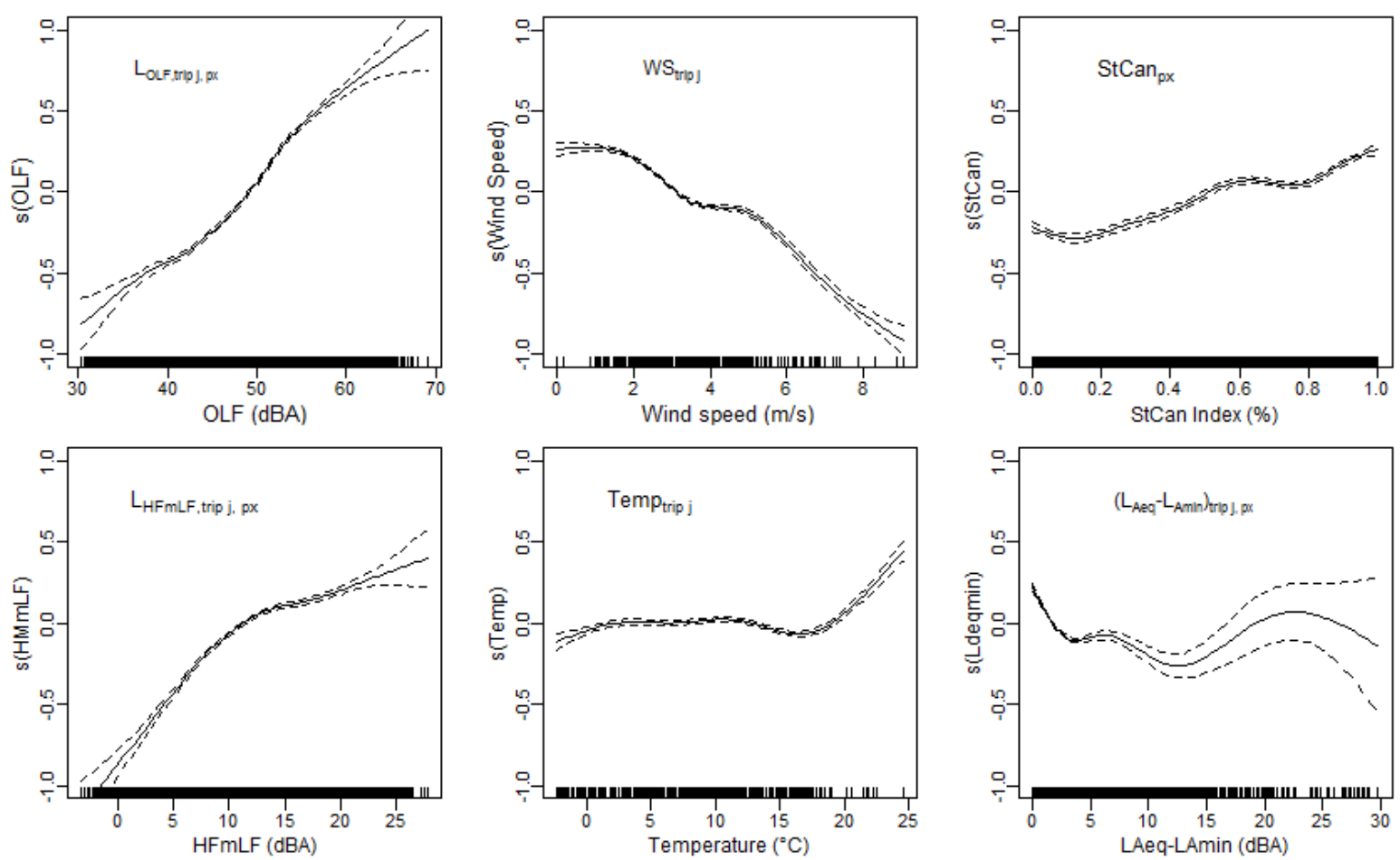

Figure 1: Splines of the six covariates of the $\mathrm{BC}_{\text {loc }}$ GAM model, ordered by strength within the model (top-left to bottom-right).
The $\mathrm{BC}_{\text {raw }}$ model shows a slightly higher deviance explained than the $\mathrm{BC}_{\text {loc }}$ model (Table 1 ). The $\mathrm{BC}_{\text {raw }}$ model also has a higher intercept. Wind speed is the strongest component. The low frequency noise level LoLf and temperature have a similar strength.

\section{Table 1: Comparing the results of the $\mathrm{BC}_{\text {loc }}$ and $\mathrm{BC}_{\text {raw }}$ models, F-value and $\mathrm{p}$-value.}

\subsection{Aggregated $\mathrm{BC}_{\text {loc }}$ and $\mathrm{BC}_{\text {raw }}$ models}

The aggregated models are built including respectively 3, 4 and 5 parameters. The temperature is not included since it proved to be of little relevance in the $\mathrm{BC}_{\text {loc }}$ model. In Table 2 the results of the aggregated models are assembled, including the number of unique combinations of the classified parameters. Again similar changes between the $\mathrm{BC}_{\mathrm{raw}, \mathrm{Xp}}$ and $\mathrm{BC}_{\mathrm{loc}, \mathrm{Xp}}$ models can be detected (were $\mathrm{X}$ is the number of aggregation 
parameters). In Figure 2 the splines of the five parameter model $\mathrm{BC}_{\mathrm{loc}, 5 \mathrm{p}}$ are shown. The deviance explained is higher and the splines are smoother compared to the $\mathrm{BC}_{\text {loc }}$ model due to the aggregation process. In the 3 parameter model $\mathrm{L}_{\mathrm{OLF}}$ the only acoustic parameter describing the source has the strongest importance in the model, but as other traffic descriptors, $\mathrm{L}_{\mathrm{HFmLF}}$ and $\left(\mathrm{L}_{\mathrm{Aeq}}-\mathrm{L}_{\mathrm{Amin}}\right)$ are added, wind speed becomes the most important covariate.
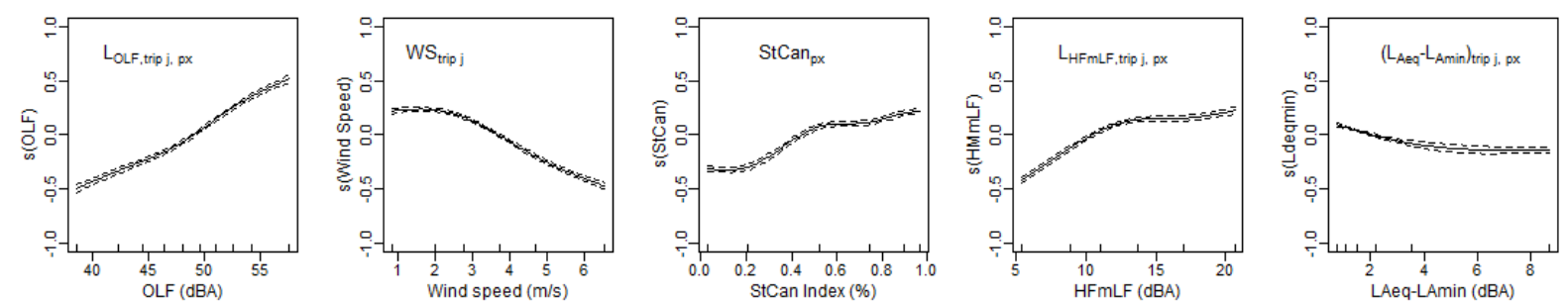

Figure 2: Splines of the five covariates of the $\mathrm{GAM} \mathrm{BC}_{\mathrm{loc}, 5 \mathrm{p}}$ model.

\subsection{Comparing the fit of the models}

The $\mathrm{BC}$ exposure during individual trips is reconstructed based on the models presented above. The results for the $\mathrm{BC}_{\mathrm{bg}}, \mathrm{BC}_{\mathrm{loc}}$ and $\mathrm{BC}_{\text {raw }}$ models are shown in Figure 3. The second row shows the results of the $\mathrm{BC}_{\text {loc }}$ model, and the third row the results of the $\mathrm{BC}_{\text {raw }}$ model. Each point in de charts represents a single trip. The $\mathrm{x}$-axis shows the measured $\mathrm{BC}$ exposure, the $\mathrm{y}$-axis the model outcome. The first column shows the total trip exposure (as $\mathrm{ng} / \mathrm{m}^{3}$ * seconds), the second column the average exposure over the trip. Both are relevant, but the correlation in the first column is strongly influenced by the duration of the trip. The predictive quality of the models is best determined by the ability to predict the trip averaged exposure. In each of these charts, the diagonal (red) and the linear fit (green) on the results are shown and the correlation and the spearman's correlation between model and measurement are given. The properties (intercept and slope) of the linear fit are shown to evaluate the model fits. The third column shows the distribution of the relative prediction: total prediction which is identical for the total and averaged evaluation. 

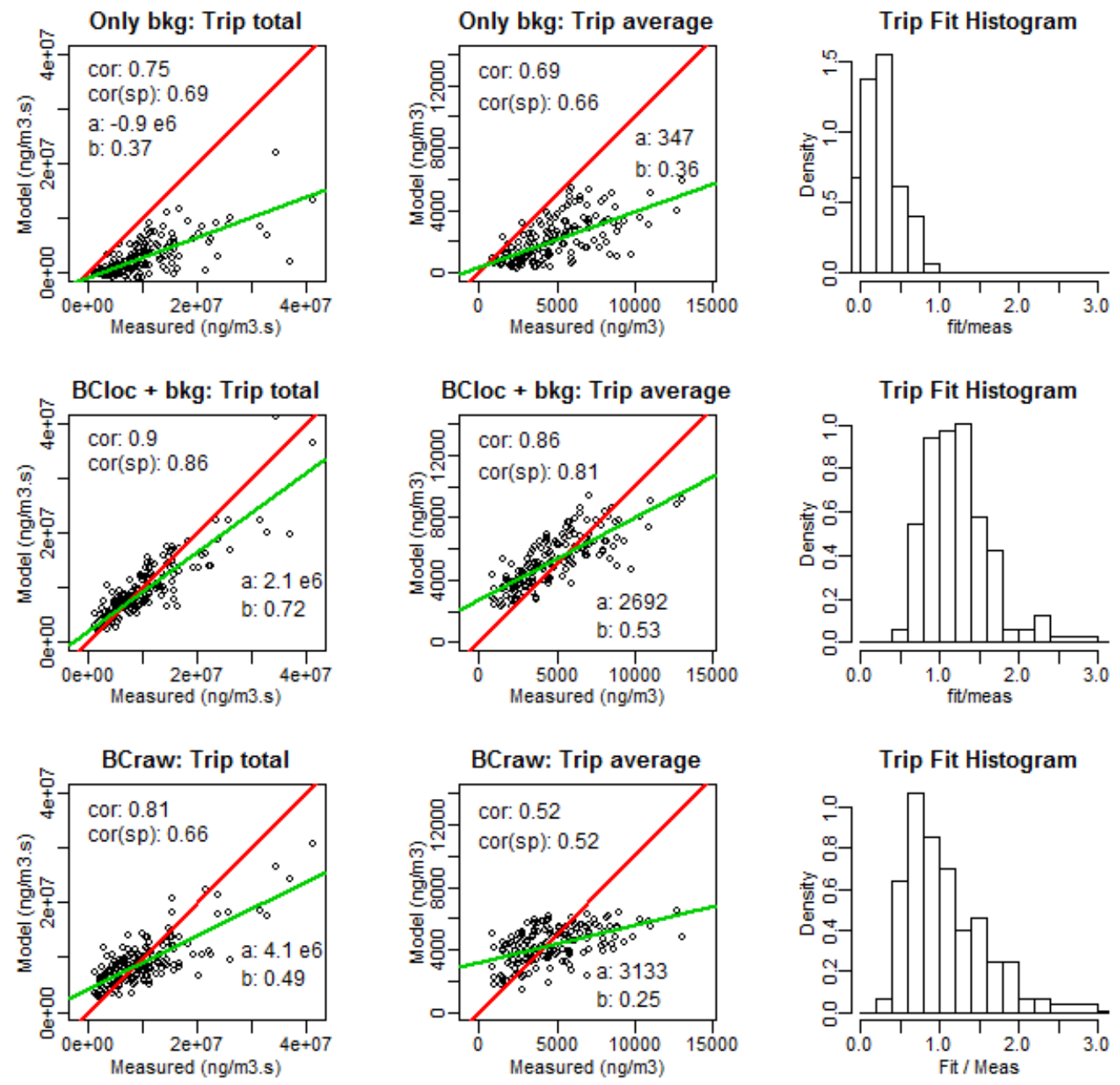

Figure 3: Evaluation of the model fit of the $\mathrm{BC}_{\mathrm{bg}}, \mathrm{BC}_{\text {loc }}$ and $\mathrm{BC}_{\mathrm{raw}}$ models. Each point in de charts 246 represents a single trip. The first row shows the results of the $\mathrm{BC}_{\mathrm{bg}}$ model, the second row the results of the $\mathrm{BC}_{\text {loc }}$ model, the third row the results of the $\mathrm{BC}_{\text {raw }}$ model. 

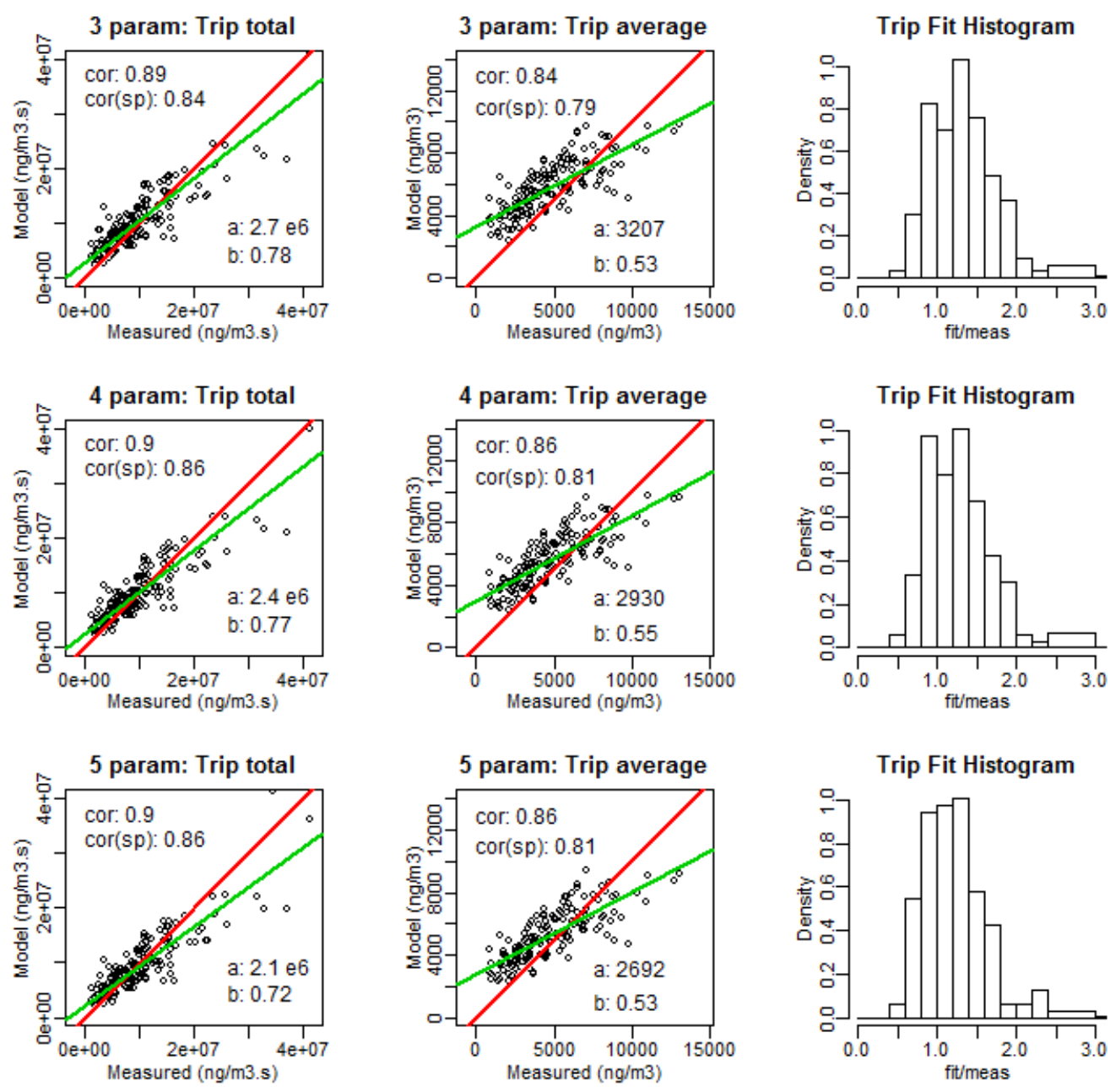

Figure 4: Evaluation of the model fit of the aggregated $\mathrm{BC}_{\mathrm{loc}}$ models for 3, 4 and 5 parameters.

A similar procedure is performed for the aggregated models. For each passage at an aggregation point the corresponding classification is used to estimate BC exposure. Summing all results over the full trajectory reconstructs the total trip exposure. In Figure 4 the fitting properties of the reconstructed trips of the $\mathrm{BC}_{\mathrm{loc}, \mathrm{Xp}}$ models are shown for the three aggregated models on $\mathrm{BC}_{\text {loc }}$. The aggregated $\mathrm{BC}_{\text {raw }}$ model evaluations are not shown.

\subsection{Comparing the predictive strength of the models}

The predictive strength of the models is checked by building variants of the models based on a random subset of $75 \%$ of the available trips and then predicting the remaining trips. This procedure is repeated 50 times. For each trip, a set of approximately 13 predictions for different model variants are thus available. The 
average $\mathrm{BC}$ trip exposure is calculated for the trips used to build the model (referred to as 'fitted trips') and predicted for the trips left out of the model (referred to as 'predicted trips'). The Pearson correlation of the fitted trips versus the measurements and the predicted tips versus the measurements are calculated for each model variant. The distributions of the correlations of the fitted trips of the model variants and the distributions of correlations of predicted trips out of the model variants are shown by model type in Figure 5. The mean correlations of the total exposure of the fitted trips for the models (as ordered in Figure 5) are $0.90,0.86,0.88,0.89$ and 0.89 , the mean correlations of the total exposure of the predicted trips are 0.88 , $0.84,0.89,0.90$ and 0.90 . The mean correlations of the averaged exposure of the fitted trips for the models are $0.78,0.71,0.75,0.77$ and 0.77 ; the mean correlations of the averaged exposure of the predicted trips are 0.75 , $0.67,0.75,0.78$ and 0.74 . Models $\mathrm{BC}_{\mathrm{loc}}, \mathrm{BC}_{\mathrm{loc}, 4 \mathrm{p}}$ and $\mathrm{BC}_{\text {loc, }, 5 \mathrm{p}}$ have similar distributions for both the fitted trips and the predicted trips. The predicted trips show wider distributions compared to the fitted trips. $\mathrm{BC}_{\text {raw }}$ and $\mathrm{BC}_{\text {loc,3p }}$ perform significantly lower than $\mathrm{BC}_{\mathrm{loc}}, \mathrm{BC}_{\mathrm{loc}, 4 \mathrm{p}}$ and $\mathrm{BC}_{\mathrm{loc}, 5 \mathrm{p}}$. The correlation of the predicted trips of $\mathrm{BC}_{\text {raw }}$ model is extremely sensitive to the trip selection. Figure $5 \mathrm{C}$ shows the relation between the correlations of the fitted and predicted trips for the individual model variants. Correlations of the predicted trips are not necessarily lower than the fitted trips. The correlation of the predictions can be higher than the correlation of the fitted trips, especially for the $\mathrm{BC}_{\mathrm{loc}}$ and $\mathrm{BC}_{\mathrm{loc}, \mathrm{xp}}$ models (Figure $5 \mathrm{C}$ ).
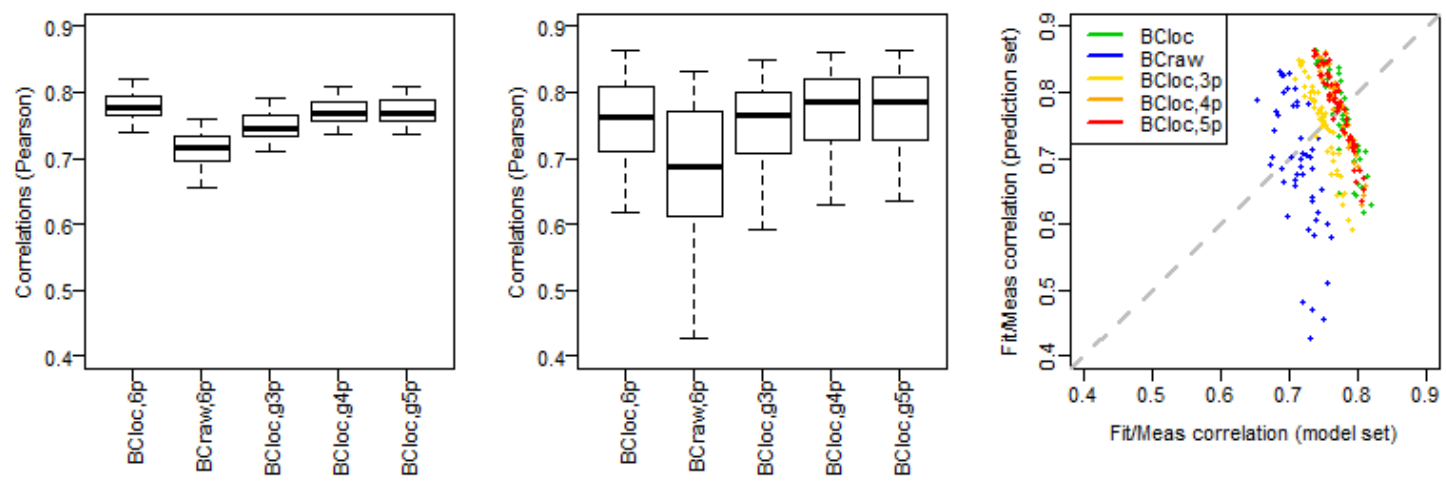

Figure 5: Distributions of the model variants correlation of the fitted trips (A), distributions of the model variants correlation of the predicted trips (B) and the relations between the correlation of the fitted trips and the correlation of the predicted trips for the individual model variants (C).

For each set of predictions of a single trip obtained from a model variant, the average prediction and the standard deviation of the trip predictions is calculated. The relative prediction (average of the total trip predictions divided by the total measured for trip j) and the standard deviation of the trip $\mathrm{j}$ predictions divided by the trip $\mathrm{j}$ total exposure are used to compare the predictive strength and sensitivity of the model 
variants to the random sampling strategy. The results are shown in two charts in Figure 6. The $\mathrm{BC}_{\text {loc }}$ model is slightly underestimating the exposure, $\mathrm{BC}_{\text {raw }}$ and $\mathrm{BC}_{\text {loc, } 3 \mathrm{p}}$ are overestimating, the $\mathrm{BC}_{\text {loc, } 4 \mathrm{p}}$ and $\mathrm{BC}_{\text {loc,5p }}$ models are centered around 1.0. The interquartile range of the distribution of the relative trip predictions for the $\mathrm{BC}_{\text {loc }}$ model is 0.38 , the ranges are slightly larger for the $\mathrm{BC}_{\mathrm{loc}, 4 \mathrm{p}}$ and $\mathrm{BC}_{\mathrm{loc}, 5 \mathrm{p}}$ models, respectively 0.47 and 0.43 . The interquartile range of the $\mathrm{BC}_{\text {loc }}$ model is 3.1 , the $\mathrm{BC}_{\text {raw }}$ model performs with 5.8 much worse. The best IQR is found for the $\mathrm{BC}_{\mathrm{loc}, 5 \mathrm{p}}$ model with 1.8. All aggregated models perform better than the $\mathrm{BC}_{\text {loc }}$ model for the distribution of the standard deviation of the trip predictions.
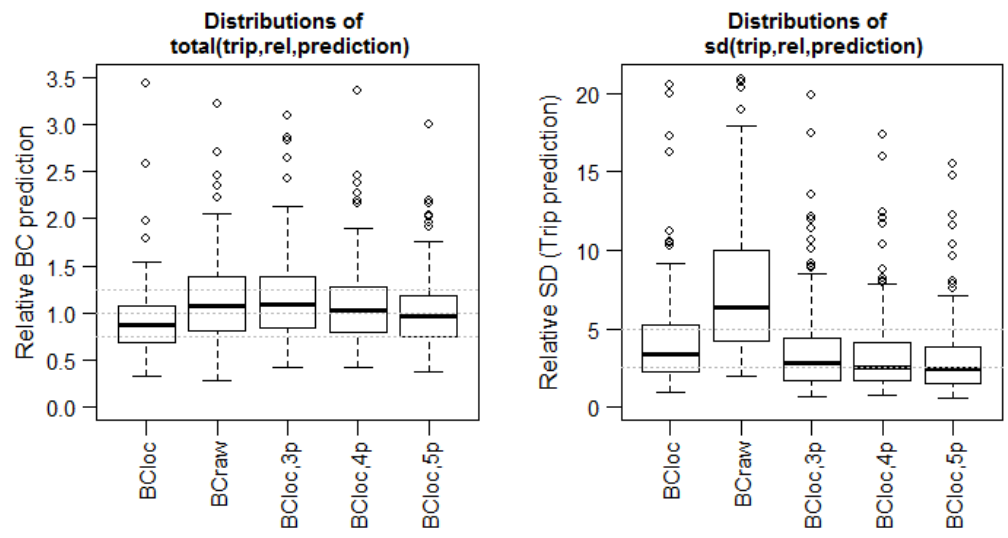

Figure 6: Distribution of the average of the relative trip predictions (A) and the distribution of the standard deviations of the trip prediction relative to the trip total measured $B C$ (B), presented by model.

\section{DISCUSSION}

The first research question investigates whether a prediction model for BC exposure could be improved by separating out long term variations in the background concentration; the second research question the whether the instantaneous local Black Carbon exposure could be predicted on the basis of local noise exposure and meteorological conditions. The first research question is addressed by comparing $\mathrm{BC}_{\mathrm{bg}}, \mathrm{BC}_{\mathrm{loc}}$ and $\mathrm{BC}_{\text {raw }}$ models. The second research question is addressed by evaluating the features of $\mathrm{BC}_{\text {loc }}, \mathrm{BC}_{\text {loc,3p, }}$ $\mathrm{BC}_{\mathrm{loc}, 4 \mathrm{p},} \mathrm{BC}_{\mathrm{loc}, 5 \mathrm{p}}$ models.

The $\mathrm{BC}_{\mathrm{bg}}$ model accounts on average for $30-40 \%$ of the total trip exposure only, but the slope in the relation between modeled and measured exposure is as large as 0.36 (Figure 3). High average trip exposure is partially related to higher background concentrations. Although the $\mathrm{BC}_{\text {raw }}$ model on average results in a good estimate of the average exposure during cycling trips, the trend line connecting prediction to measurement (Figure 3) slopes at 0.25 . The strong influence of wind speed and temperature indicates that this model 
mainly tries to resolve the temporal variations in the background contributions and fails to include indicators for local exposure. Integrating the $\mathrm{GAM}$ model for $\mathrm{BC}_{\mathrm{loc}}$ with measured $\mathrm{BC}_{\mathrm{bg}}$ combines best of both worlds: temporal variability of the $\mathrm{BC}_{\mathrm{bg}}$ with spatial variability of the $\mathrm{BC}_{\mathrm{loc}}$ model and results in a slope between modeled and measured average trip exposure of 0.53 .

A similar conclusion can be drawn when evaluating the predictive power of the models in Section 3.4. The wide distribution of the correlations of the predicted trips in the model variants of the $\mathrm{BC}_{\text {raw }}$ model (Figure 5B) and the high relative standard deviation of the individual trip prediction (Figure 6B) illustrate the sensitivity of the $\mathrm{BC}_{\text {raw }}$ model to the trip selection. All variants of the $\mathrm{BC}_{\text {loc }}$ model perform significantly better. The $\mathrm{BC}_{\text {loc }}$ model is lightly sensitive to over fitting for the low exposure values, which reflects in the underestimation of the relative trip prediction (Figure 6A).

As it is now established that the $\mathrm{BC}_{\text {loc }}$ models outperform models that aim at predicting the whole exposure at once, let us now look in detail at the choice of predictive variables for these models. In the $\mathrm{BC}_{\text {loc }}$ model $L_{O L F, p_{x}, j}$ is the strongest component and wind speed the second strongest. The negligible importance of temperature in the model can be explained as follows. Temperature is expected to affect emission and is an indicator for meteorological conditions that influence dispersion. The background concentration indeed shows a distinctive seasonal pattern and temperature is related to season (see supplementary data 1.9 figure S3). $\mathrm{BC}_{\mathrm{bg}}$ seems to include the temperature dependence sufficiently thereby reducing the relevance of temperature in the $\mathrm{BC}_{\text {loc }}$ model. Wind speed has a similar relationship with background concentration as temperature (supplementary data 1.9 figure S4). The strength of the wind speed covariate is therefore also reduced in the $\mathrm{BC}_{\mathrm{loc}}$ compared to its strength in the $\mathrm{BC}_{\text {raw }}$ model but in contrast to the temperature, it is still highly relevant. This is explained by the observation that wind speed not only influences background concentrations but also instantaneous dispersion of the local emissions. Higher wind speeds reduce the local exposure of cyclists. StCAN and $\mathrm{LHFmLF}_{\mathrm{H}}$ are also stronger in the $\mathrm{BC}_{\text {loc }}$ model compared to the $\mathrm{BC}_{\text {raw }}$ model probably because major confounders are eliminated by treating $\mathrm{BC}_{\mathrm{bg}}$ separately. The $\mathrm{BC}_{\mathrm{loc}}$ model becomes more sensitive to spatially varying variables such as vehicle speed and local air pollution accumulation effect. The latter effects and its dependence on street canyon geometry (measured here as StCAN) is confirmed by physical calculations of street canyon accumulation, validated with measurements such as the one presented by Berkowicz et al (2008). 
High-degree-of-freedom models such as the GAM model risk to over fit the data that they are based on and

while losing the ability to generalize to other situations. This can be prevented by reducing the number of input variables (covariates) and -in the particular case of GAM models - also by reducing the number of values that the variable can take. Classification of the covariates indeed reduces the degrees of freedom (Table 2) in the model. Because of the change in number of data points, the goodness-of-fit parameters and the strength of the covariates in the models cannot be compared directly, the quality of the models is determined by evaluating the properties of the predictions. In addition, a lower number of variables reduces the complexity of the model and simplifies implementation.

Turning to the model prediction evaluation shown in Figure 5 and Figure 6, it is clear that the $\mathrm{BC}_{\text {loc,g3p }}$ model performs worse than the $\mathrm{BC}_{\text {loc }}$ model in predicting the average exposure. This is not unexpected as the information used in the prediction is significantly reduced. The reduction in number of covariates and the clustering of values is expected to make the model less sensitive to trip sampling in the model variants and to make it should perform better on the independent test data. For $\mathrm{BC}_{\text {loc,g3p }}$ the aggregation is to strong to observe this positive effect. The $\mathrm{BC}_{\text {loc,g4p }}$ and $\mathrm{BC}_{\text {loc,g5p }}$ models slightly outperform the $\mathrm{BC}_{\text {loc }}$ model when evaluated for correlation between model and measurement on the data not used for fitting (Figure 5B). The distribution of relative trip predictions (Figure $6 \mathrm{~A}$ ) is wider for the $\mathrm{BC}_{\text {loc,g4p }}$ and $\mathrm{BC}_{\text {loc,g5p }}$ models compared to the $\mathrm{BC}_{\text {loc }}$ model, but the distributions are centered around 1 . The relative standard deviation of the individual trip prediction (Figure $6 \mathrm{~B}$ ) is slightly better. The $\mathrm{BC}_{\text {loc,g5p }}$ outperforms the $\mathrm{BC}_{\text {loc }}$ model despite the fact that it only reduces the number of data points from 37722 to 8832 . The aggregation process removes the lowest values in the basic dataset, resulting in a better prediction of the total trip $\mathrm{BC}$ exposure.

It is more difficult to distinguish between the $\mathrm{BC}_{\text {loc,g4p }}$ and $\mathrm{BC}_{\text {loc,g5p }}$ models. The only relevant difference is the reduction of the intercept in the $\mathrm{BC}_{\text {loc,g5p }}$ model. The short term dynamics $\left(\mathrm{L}_{\mathrm{Aeq}}-\mathrm{L}_{\mathrm{Amin}}\right.$ ) covariate - the variable differentiating $\mathrm{BC}_{\text {loc,g5p }}$ from $\mathrm{BC}_{\text {loc,g4p }}$ - distinguishes between two different traffic conditions: low values can be linked to situations of congested traffic, many cars with constant noise emission (idling) result in constant noise levels; high values indicate short distinct events, typically a single car passing by at a relatively high speed. This last traffic situation is however rare during the rush hour and is under represented in our (rush hour only) database. When similar measurements would be performed outside the rush hour, 
this covariate might prove more relevant. With the available measurements it cannot conclude that the $\mathrm{BC}_{\text {loc }, 5 \mathrm{p}}$ model is better than $\mathrm{BC}_{\text {loc, } 4 \mathrm{p}}$ model.

In general, the simplification of the model improves its quality as long as the 4 or 5 most significant parameters are kept. The models suggested in this paper perform well mainly due to the unique choice of noise indicators that are directly related to relevant traffic dynamics. The LoLF covariate detects traffic volume including acceleration both linked to higher particulate emissions. $\mathrm{L}_{\mathrm{HFmLF}}$ indicates the traffic speed; emissions increase with speed at low speeds, but saturate at higher speeds because constant high speed result in more efficient combustion processes and lower particulate emissions. Similar relationships were reported for nanoparticles in Uhrner et al., 2011 and for gaseous emissions in De Vlieger et al., 2000. This also explains why models based on $\mathrm{L}_{\text {Aeq }}$ are less successful: $\mathrm{L}_{\text {Aeq }}$ is related to human loudness perception and does allow distinguishing situations with different traffic dynamics (Boogaard et al., 2008). Ross et al., 2011 relates a spectral noise evaluation to air pollution on a fixed monitoring station. Although these measurements are not directly comparable with the measurements in this paper, the same frequency bands are found to be relevant.

\section{CONCLUSIONS}

This paper proves that it is possible to predict instantaneous $\mathrm{BC}$ concentrations based on mobile noise measurements. The in-traffic exposure to Black Carbon of bicyclists is determined by background concentration, distance to source, local traffic density and speed, local traffic dynamics, local street geometry, and meteorological conditions. Spectral evaluation of (mobile) noise measurements can be used to implicitly detect local traffic conditions directly related to the local BC emission. Predicting personal BC exposure of cyclists proved only successful after splitting the model into a background contribution and a local component. In particular, it was shown that the spatial variability due to the local traffic contribution can be modeled using four parameters: the low frequency noise LoLf related to the traffic volume and engine throttle, the difference between high and low frequencies $\mathrm{L}_{\mathrm{HFmLF}}$ related to the traffic speed, the instantaneous wind speed and the street canyon index both related to the local accumulation of the $\mathrm{BC}$. The overall trip exposure is predicted by the four categorized parameters $\mathrm{GAM}$ model, $\mathrm{BC}_{\text {loc,g4p }}$, with a correlation of 0.90 . The average trip exposure is predicted with a correlation of 0.78 . 
The structure of the model presented in this paper can be expected to be valid for different areas in the world since parameters are chosen with a physical background in mind and because the model has been well validated. Since fleet composition and driving behavior might differ between different parts of the world, it is suggested that each mobile noise measurement campaign is accompanied with a partial BC sampling to reestablish the model coefficients. The measurement underlying the specific model presented here, are performed in a typical (European) environment, a medium size city including suburbs and green areas, representative for the living environment of the majority of the population in Europe. The exact same model including model coefficients is expected to be applicable in any city sufficiently similar to this situation.

Mobile noise measurements on bicycles have the potential to provide the spatial detail and high temporal resolution that is necessary to predict the urban exposure to black carbon, including the local effects of route choice. Mobile noise measurements therefore have the power to replace large scale in-traffic personal air pollution exposure measurements and can be performed on larger populations at a significantly lower cost than traditional participatory sampling techniques. In this way the results obtained in this study could be useful for raising public awareness, changing personal behavior by selecting low exposure routes and to perform large scale epidemiological research on the impact of personal BC exposure on health.

Berghmans, P.; Bleux, N.; Int Panis, L.; Mishra, V. K.; Torfs, R.; Van Poppel, M., Exposure assessment of a cyclist to PM(10) and ultrafine particles. Science of the Total Environment 2009, 407, (4), 1286-1298.

Boogaard, H.;Borgman F.;Kamminga J.; Hoek G.; Exposure to ultrafine and fine particles and noise during cycling and driving in 11 Dutch cities. Atmosferic environment 43 (2009) 4234-4242.

Berkowicz R., Ketzel M., Jensena S.S, Hvidberg M., Raaschou-Nielsen O., Evaluation and application of OSPM for traffic pollution assessment for a large number of street locations, Environmental Modelling \& Software 2008, 296-303.

Can, A.; Botteldooren, D., Towards Traffic Situation Noise Emission Models. Acta Acustica United with Acustica 2011, 97, (5), 900-903. 
Can, A.; Leclercq, L.; Lelong, J.; Botteldooren, D., Traffic noise spectrum analysis: Dynamic modeling vs. experimental observations. Applied Acoustics 2010, 71, (8), 764-770.

de Hartog, J. J.; Boogaard, H.; Nijland, H.; Hoek, G., Do the health benefits of cycling outweigh the risks? Environ Health Perspect. 2010 August; 118(8): 1109-1116.

de Nazelle, A.; Fruin, S.; Westerdahl, D.; Martinez, D.; Matamala, J.; Kubesch, N.; Nieuwenhuijsen, M., Traffic Exposures and Inhalations of Barcelona Commuters. Epidemiology 2011, 22, (1), S77-S78.

Dekoninck, L.; Botteldooren, D.; Int Panis, L., Guidelines for participatory noise sensing based on analysis of high quality mobile noise measurements. Internoise 2012 (conference), 394-402

De Vlieger I., D De Keukeleere D., Kretzschmar J.G., Environmental effects of driving behaviour and congestion related to passenger cars, Atmospheric Environment, Volume 34, Issue 27, 2000, Pages 4649-4655

Dominici, F.; McDermott, A.; Zeger, S. L.; Samet, J. M., On the use of generalized additive models in time-series studies of air pollution and health. American Journal of Epidemiology 2002, 156, (3), 193-203.

Dons, E.; Int Panis, L.; Van Poppel, M.; Theunis, J.; Willems, H.; Torfs, R.; Wets, G., Impact of time-activity patterns on personal exposure to black carbon. Atmospheric Environment 2011, 45, (21), 3594-3602.

Dons, E.; Int Panis, L.; Van Poppel, M.; Theunis, J.; Wets, G., Personal exposure to Black Carbon in transport microenvironments. Atmospheric Environment 2012, 55.

Eisenman, S. B.; Miluzzo, E.; Lane, N. D.; Peterson, R. A.; Ahn, G.-S.; Campbell, A. T., BikeNet: A Mobile Sensing System for Cyclist Experience Mapping. Acm Transactions on Sensor Networks 2009, 6, (1).

Foraster, M.; Deltell, A.; Basagana, X.; Medina-Ramon, M.; Aguilera, I.; Bouso, L.; Grau, M.; Phuleria, H. C.; Rivera, M.; Slama, R.; Sunyer, J.; Targa, J.; Kunzli, N., Local determinants of road traffic noise levels versus determinants of air pollution levels in a Mediterranean city. Environmental Research 2011, 111, (1), 177-183.

Int Panis, L.; de Geus, B.; Vandenbulcke, G.; Willems, H.; Degraeuwe, B.; Bleux, N.; Mishra, V.; Thomas, I.; Meeusen, R., Exposure to particulate matter in traffic: A comparison of cyclists and car passengers. Atmospheric Environment 2010, 44, (19), 2263-2270.

Isakov, V., J. S. Touma, et al. (2009). "Combining Regional- and Local-Scale Air Quality Models with Exposure Models for Use in Environmental Health Studies." Journal of the Air \& Waste Management Association 59(4): 461-472. 
Kanjo, E., NoiseSPY: A Real-Time Mobile Phone Platform for Urban Noise Monitoring and Mapping. Mobile Networks \& Applications 2010, 15, (4), 562-574.

Maisonneuve, N.; Stevens, M.; Niessen, M. E.; Steels, L., NoiseTube: Measuring and mapping noise pollution with mobile phones. Information Technologies in Environmental Engineering 2009, 215-228.

Pearce, J. L.; Beringer, J.; Nicholls, N.; Hyndman, R. J.; Tapper, N. J., Quantifying the influence of local meteorology on air quality using generalized additive models. Atmospheric Environment 2011, 45, (6), 1328-1336.

Ross Z., Kheirbek I., Clougherty J., Ito K., Matte T., Markowitz S., Eisl H., Noise, air pollutants and traffic: Continuous measurement and correlation at a high-traffic location in New York City. Environmental Research, 2011, 1054-1063.

Uhrner, U., M. Zallinger, et al. (2011). "Volatile Nanoparticle Formation and Growth within a Diluting Diesel Car Exhaust." Journal of the Air \& Waste Management Association 61(4): 399-408

von Klot, S.; Cyrys, J.; Hoek, G.; Kuhnel, B.; Pitz, M.; Kuhn, U.; Kuch, B.; Meisinger, C.; Hormann, A.; Wichmann, H. E.; Peters, A., Estimated Personal Soot Exposure Is Associated With Acute Myocardial Infarction Onset in a Case-Crossover Study. Progress in Cardiovascular Diseases 2011, 53, (5), 361-368.

WHO Europe, 2012: Health effects of black carbon, ISBN: 9789289002653.

Wood, S. N., On confidence intervals for generalized additive models based on penalized regression splines. Australian \& New Zealand Journal of Statistics 2006, 48, (4). 